\title{
Quality of solo ambulance care by physician assistants versus ambulance nurses for non-conveyed patients in the
}

\section{Netherlands: An observational study [version 1; peer review: 1}

\section{approved with reservations]}

\author{
Lilian Vloet ${ }^{1,2}$, Daniël Winterink ${ }^{3}$, Aico Gerritsen ${ }^{3}$, Wim Heutz ${ }^{3}$, \\ Thijs van Zonneveld ${ }^{1}$, Sivera Berben ${ }^{1,2}$, Remco Ebben (iD)1
}

\footnotetext{
${ }^{1}$ Research department of emergency and critical care, HAN University of Applied Sciences, Nijmegen, Gelderland, 6525 EN, The Netherlands

${ }^{2}$ Radboud Institute for Health Sciences IQ healthcare, Radboud University Medical Center, Nijmegen, 6525 EZ, The Netherlands

${ }^{3}$ Emergency Medical Service Gelderland-Midden, Veiligheidsregio Gelderland-Midden, Arnhem, $6828 \mathrm{HZ}$, The Netherlands
}

V1 First published: 07 May 2021, 10:357
https://doi.org/10.12688/f1000research.51745.1
Latest published: 07 May 2021, 10:357

https://doi.org/10.12688/f1000research.51745.1

\section{Abstract}

Background

The aim of this study was to compare the ambulance care process, follow-up care and patient experience between physician assistants and ambulance nurses operating as solo ambulance care providers, for a non-conveyed patient population.

\section{Methods}

An observational design was used. Characteristics of patients and events and the care process were retrieved from the ambulance registration database. Data on follow-up care and patient experience were collected through questionnaires.

\section{$\underline{\text { Results }}$}

Of the included solo ambulance events, 49/379 (12.9\%) were performed by physician assistants, 330/379 (87.1\%) were performed by ambulance nurses. For initial complaints and the on-scene diagnoses there were no significant differences between the physician assistants and ambulance nurses. 90/165 (54.5\%) of the patients requested follow-up care after being non-conveyed with no significant association between the PA and ambulance nurse group $(p=.293)$. For type of follow-up care, $91.9 \%$ of the follow-up emergency care requests in this study came from patients treated by an ambulance nurse. There were no significant differences in patients' experience for clinician attitude and behavior, treatment, and communication between physician assistants and ambulance nurses, although pain

\section{Open Peer Review}

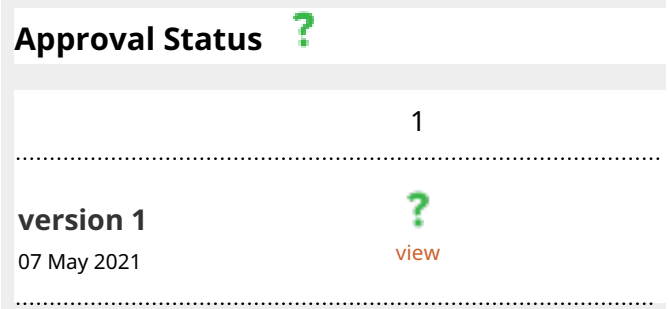

1. Erika Frischknecht Christensen (ID), Aalborg University Hospital, Aalborg, Denmark Any reports and responses or comments on the article can be found at the end of the article. 
management and explanation about the non-conveyance decision could be improved

\section{Conclusions}

Besides small statistical but not clinically significant differences, this study indicated comparable solo ambulance care provided by a physician assistant or an ambulance nurse concerning the care process, follow-up care and patient experience. Patients treated by physician assistants seek less follow-up emergency care after nonconveyance, compared to ambulance nurses. Overall, patients experienced good attitude and behavior, treatment, and communication of the solo ambulance professional. Future well powered studies to gain insight in effects of PAs in ambulance care are needed, as well as studies in which PAs apply all additional skills they are licensed to.

\section{Keywords}

Emergency Medical Services [MeSH], Quality of Healthcare [MeSH], Physician Assistants [MeSH], non-conveyance

Corresponding author: Remco Ebben (remco.ebben@han.nl)

Author roles: Vloet L: Conceptualization, Formal Analysis, Methodology, Supervision, Writing - Original Draft Preparation, Writing Review \& Editing; Winterink D: Conceptualization, Formal Analysis, Investigation, Methodology, Project Administration, Visualization, Writing - Review \& Editing; Gerritsen A: Conceptualization, Methodology, Writing - Review \& Editing; Heutz W: Conceptualization, Methodology, Writing - Review \& Editing; van Zonneveld T: Formal Analysis, Investigation, Project Administration, Visualization, Writing - Review \& Editing; Berben S: Supervision, Writing - Original Draft Preparation, Writing - Review \& Editing; Ebben R: Conceptualization, Formal Analysis, Investigation, Methodology, Project Administration, Supervision, Validation, Visualization, Writing - Original Draft Preparation, Writing - Review \& Editing

Competing interests: No competing interests were disclosed.

Grant information: The author(s) declared that no grants were involved in supporting this work.

Copyright: (c) 2021 Vloet L et al. This is an open access article distributed under the terms of the Creative Commons Attribution License, which permits unrestricted use, distribution, and reproduction in any medium, provided the original work is properly cited.

How to cite this article: Vloet L, Winterink D, Gerritsen A et al. Quality of solo ambulance care by physician assistants versus ambulance nurses for non-conveyed patients in the Netherlands: An observational study [version 1; peer review: 1 approved with reservations] F1000Research 2021, 10:357 https://doi.org/10.12688/f1000research.51745.1

First published: 07 May 2021, 10:357 https://doi.org/10.12688/f1000research.51745.1 


\section{Introduction}

Emergency medical services (EMS) face a challenging environment with an increasing number of ambulance deployments ${ }^{1,2}$. Reasons for this increase are a growing population with more complex healthcare problems and comorbidities, repeated requests for ambulance care, and the request of ambulance care for primary care problems $\mathrm{s}^{3-6}$. Within this increasing demand, there is also a growing population that receives ambulance care without conveyance to another healthcare facility. Non-conveyance is defined as an ambulance dispatched with on-scene assessment and treatment, but without conveyance to a hospital ${ }^{7,8}$. The non-conveyance decision can be initiated by the ambulance professional (sometimes after consultation with a general practitioner or medical specialist) or the patient and/or their relatives. For general patient populations the non-conveyance proportion ranges from 3.7-93.7\% . Although the proportion of non-conveyance is increasing, ambulance care professionals experience the conveyance decision making process as difficult, challenging and often unsupported by guidelines ${ }^{10,11}$. Patients are mostly satisfied with provided care in non-conveyance situations, but non-conveyance evokes fear, shame, and a need for reassurance ${ }^{12,13}$.

To meet the increased demand and patient complexity while securing patient safety and effectiveness in ambulance care, stratification of ambulance care using different types of ambulance care professionals is needed. Therefore, physician assistants (PA's) were introduced in ambulance care in the Netherlands. Primary rationale for the introduction is to provide efficient and adequate care by different types of ambulance care professionals to meet the growing demand. Secondarily, to retain ambulance nurses EMSs want to offer ambulance nurses a career perspective at a master's level, such as the nurse practitioner or physician assistant roles ${ }^{14}$.

Despite the introduction of the PA in ambulance care, little is known about the effects. A recent systematic review showed limited available evidence on the introduction of master's level educated professionals in ambulance care, with lacking evidence on patient outcomes ${ }^{15}$. This underpins the need for further research on effectiveness, efficiency, patient safety, and accessibility of the introduction of PAs in ambulance care. Within the chain of emergency care, a systematic review showed that PAs in the emergency department setting are reliable in assessment of medical complaints and performing procedures, and that PAs are accepted by patients and ED staff ${ }^{16}$.

As the effects of PAs working in prehospital ambulance care are currently unclear, the aim of this study was to compare the ambulance care process, follow-up care, and patient experience between physician assistants and ambulance nurses operating as solo ambulance care providers, for a nonconveyed patient population.

\section{Methods}

\section{Design}

This study had an observational design and is reported using the STROBE-statement ${ }^{17}$. Ethical approval was obtained from the Ethical Research Committee of the HAN University of Applied Sciences (see ethical statement below).

\section{Setting}

Ambulance care in the Netherlands is dispatched through the dispatch center and can be requested via the national emergency number or by healthcare professionals (such as general practitioners). After triage, ambulance care can be dispatched with urgency level A1 (arrival $<15$ minutes), A2 (arrival $<30$ minutes, and $\mathrm{B}$ (planned ambulance care). The dispatch center can dispatch a fully equipped ambulance with conveyance facilities (one driver and one ambulance nurse) or a solo ambulance unit. The non-nurse driver at the fully equipped ambulance has a supporting role to the ambulance nurse during the care and transfer process. A solo ambulance unit is an ambulance vehicle with basic and advanced life support equipment but without conveyance facility ${ }^{18}$. A solo ambulance unit can be staffed by one ambulance care professional, either an ambulance nurse, physician assistant or nurse practitioner.

Traditionally, ambulances in the Netherlands are staffed by ambulance nurses. Ambulance nurses in the Netherlands are registered nurses (RN) with additional education and working experience in anesthesia, Intensive Care (IC) or emergency department care (ED), before entering the 9-month ambulance training program. After graduation from the ambulance training program, ambulance nurses have functional autonomy within the framework of their national EMS protocols ${ }^{19,20}$. In comparison to ambulance nurses, The PA holds a master's degree in the medical domain, the 30 month educational program has theoretical and clinical phases, with a strong focus on diagnostic skills, such as organ tract system examination ${ }^{14,21}$. PAs have advanced diagnostic skills at master level and they are allowed by law to independently diagnose and treat patients beyond the national EMS protocol. Furthermore, they can perform autonomously advanced medical procedures, such as surgical procedures, endoscopy, punctures, elective cardioversions, and medication prescription.

This study was performed an urban EMS region in the Netherlands. This EMS uses a combination of advance life support and basic life support ambulance units, dispatch in this region is guided by the Dutch Triage Standard ${ }^{22}$. The solo ambulance care unit in this EMS is solely dispatched between 07:00h$23.00 \mathrm{~h}$ as historical data showed non-conveyance occurs more in urban areas and mostly during these hours. The solo ambulance care provider at this EMS is either an ambulance nurse or a PA. For this EMS 12 ambulance nurses and 5 PAs are eligible to staff the solo ambulance. All PAs worked as ambulance nurses before they entered the PA educational program.

In the Netherlands, ambulance nurses and PAs are eligible to apply for the solo ambulance care selection procedure when they have a minimum of 3 years working experience as ambulance nurse and hold an Advanced Medical Life Support certificate. The selection procedure consists of an interview assessment, medical assessment, and driving assessment. After passing this selection procedure, candidates undertake 
an additional 5-day driving training course with a theoretical and practical exam, and a 1-day training program aimed at (a) on-scene safety management, (b) incident management, (c) starting a resuscitation on your own, and (d) mass casualty incident coordination. Finally, they follow a 5-day traineeship with an experienced solo ambulance care professional.

\section{Population}

In the period January-July 2019 this EMS region performed 40,910 ambulance dispatches, of which 1,549 were performed by a solo ambulance unit. Each dispatch has a unique identification number that is automatically generated. From the EMS we received the unique identification numbers of all ambulance dispatches with patient contact that ended in non-conveyance, except when (a) the ambulance run was cancelled by the dispatch center in case the ambulance was no longer needed, (b) the patient was conveyed by a regular ambulance after solo ambulance attendance and assessment, (c) the solo ambulance assisted another regular ambulance, (d) the patient deceased, (e) the patient refused care, (f) the solo ambulance run performed was for stand-by purposes, and ( $g$ ) the patients' home address was not available in the ambulance registration database.

\section{Data collection}

Data was collected from 1) the ambulance registration database and 2) by questionnaires.

The ambulance registration database is an internal EMS database in which they register all data taken on an ambulance run. It can only be accessed by EMS managers and data managers, and on request for research purposes.

Characteristics of the patient (age, sex, time and day of ambulance request, and vital functions/observation scales), and the care process (EMS dispatch complaint, and initial on-scene diagnosis) were retrieved from the ambulance registration system. The initial complaint and initial on-scene diagnoses were categorized into one the of the 23 chapters of the International Statistical Classification of Diseases and Related Health Problems 10th revision (ICD-10) ${ }^{23}$. Categorization was performed by two independent researchers (DW, TvZ). In case of doubt a third researcher was consulted (RE).

To collect data on follow-up care and patient experience a questionnaire was developed. The first part of the questionnaire contained six questions about follow-up care after nonconveyance (repeated access to healthcare, relation between first EMS contact and repeated healthcare contact, reasons for repeated access to healthcare, and whether the patient received a prescription during a second healthcare contact). These questions were based on recent literature on non-conveyance ${ }^{9}$. The second part of the questionnaire focused on patient experience and was based on the validated Consumer Quality Index Emergency Ambulance Care (CQI-index) ${ }^{24}$. This CQI-index is a 48 -item validated questionnaire to measure patient experience on seven scales: emergency number and dispatch center, attitude and behavior of the ambulance professional, treatment by ambulance professional, communication by ambulance professional, non-conveyance, transportation, and emergency department. The CQI-index was validated through factor analysis, assessing Cronbach's alpha, and face validity, and the scales showed moderate to good reliability (Cronbach's alpha 0,65-0,80) ${ }^{25}$. As this study focuses on on-scene and follow-up care by a solo ambulance care unit, we only used the 13 items from the four scales attitude and behavior, treatment, communication and non-conveyance. Patients could answer on a 4-point scale ('no, not at all', 'yes, a little', 'yes, for the most part', 'yes, totally'), with an additional option for 'can't remember' or 'not applicable'. An information letter, the questionnaire, and a return envelope were sent to the included patients. The information letter contained information about the aim, anonymity, privacy, data management, informed consent, and contact information. The information letter also stated that if the patient returned the questionnaire, this was interpreted as informed consent to participate. Reminders, including the questionnaire and a return envelope, were sent after three weeks. Data from the ambulance registration database and questionnaires were linked on patient level by using the unique identification number that is automatically generated at ambulance dispatch. This linkage was performed by the EMS, the researchers only had access to an anonymized database. All variables from the ambulance registration database and questionnaire are displayed in Extended data.

\section{Data analysis}

Results are presented using descriptive statistics. To determine effects between the PA and ambulance nurse groups, Chi-square tests, Fisher's exact tests, and t-tests were performed. Statistical significance was defined as a p-value $<0.05$. Missing data were at random, all statistical tests were performed on available data, no imputation occurred. Data were analyzed using SPSS version 25.0.

\section{Results}

Of the 1,549 solo ambulance events, 379 (24.5\%) met inclusion criteria (Figure 1). Main reasons for exclusion were the patient being conveyed by an ambulance after solo ambulance assessment, and a standby ambulance run. Of the included solo ambulance events, $49 / 379$ (12.9\%) were performed by PAs, $330 / 379(87.1 \%)$ were performed by ambulance nurses.

\section{Care process}

There were more female patients non-conveyed by PAs, compared to ambulance nurses $(\mathrm{p}=.042)$ (Table 1). The average age of the whole group was 43.1 years (SD 24.8) with no differences between the PA and the ambulance nurses' group (43.2 years vs. 43 years, $\mathrm{p}=.952)$. $41.4 \%$ of the ambulance was requested between $17.00 \mathrm{~h}-23.00 \mathrm{~h}$, there was no difference between PAs and ambulance nurses $(\mathrm{p}=.233)$. For the distribution of solo ambulance care runs across days of the week there was an overall statistical difference between the PA and the ambulance nurses' groups, with no solo ambulance runs by PAs on Wednesdays $\left(\mathrm{X}^{2}=21.605, \mathrm{p}=.001\right)$.

The initial complaints and on-scene diagnoses are described in Table 2. The initial complaints $\left(\mathrm{X}^{2}=7,354, \mathrm{p}=.807\right)$ 


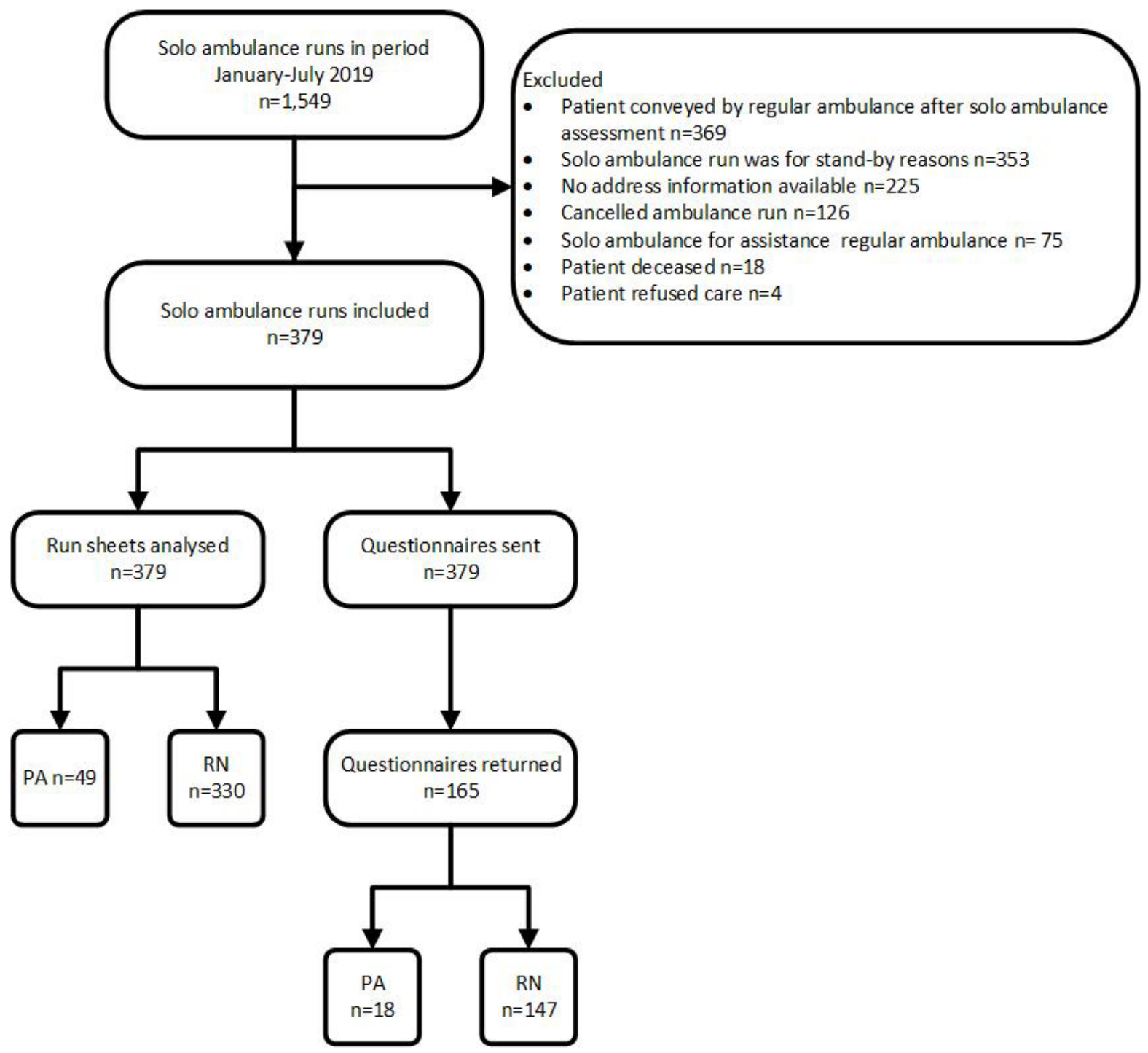

Figure 1. data collection flowchart.

and on-scene diagnoses $\left(\mathrm{X}^{2}=7,711, \mathrm{p}=.773\right)$ were similar in the PA and ambulance nurse groups. The top three initial complaints during EMS dispatch could be classified into injury, poisoning and certain other consequences of external causes; other/non classifiable; and mental, behavioral and neurodevelopmental disorders. This top three was equal for the PA and ambulance nurse group. For 278/379 (73.4\%) of the patients on-scene diagnosis were available. The three most frequent diagnoses for the total group and ambulance nurses' group could be classified into injury, poisoning and certain other consequences of external causes; other/non classifiable; and diseases of the circulatory system. For the PA group, certain infectious and parasitic diseases, diseases of the nervous system, and diseases of the circulatory system, completed the top three. Data on vital functions/observation scales largely showed comparable populations for PAs and ambulance nurses, with exception of insufficiency of breathing ( $0.3 \%$ vs. $5 \%, \mathrm{p}=.038)$ (Table 3$)$.

\section{Follow-up care}

Questionnaires to measure follow-up care and patient experience were returned by $165 / 379$ respondents (43.5\%), of which $18 / 165(10.9 \%)$ were treated by PAs and 147/165 (89.1\%) were treated by ambulance nurses (Figure 1).

A total of $90 / 165(54.5 \%)$ of the patients requested followup care after being non-conveyed by the solo ambulance care unit (Table 4). There was no significant association between follow-up care requests between the PA and ambulance nurse group $\left(\mathrm{X}^{2}=5,328, \mathrm{p}=.293\right.$ ), but $91.9 \%$ of the follow-up emergency care requests in this study came from patients treated by 
Table 1. DEMOGRAPHICS.

\begin{tabular}{|c|c|c|c|c|c|c|c|c|}
\hline \multirow[b]{2}{*}{ VARIABLES } & \multicolumn{2}{|c|}{ Total group } & \multicolumn{2}{|c|}{$\begin{array}{c}\text { Ambulance } \\
\text { nurse }\end{array}$} & \multicolumn{2}{|c|}{ PA } & \multirow[b]{2}{*}{$\begin{array}{c}\text { Point } \\
\text { estimate }\end{array}$} & \multirow[b]{2}{*}{ p-value } \\
\hline & $\mathbf{N}$ & $\%$ & $\mathbf{N}$ & $\%$ & $\mathbf{N}$ & $\%$ & & \\
\hline GENDER N (\%) & 378 & & 329 & & 49 & & $4,122^{\#}$ & $.042^{*}$ \\
\hline MALE & 190 & $(50.1 \%)$ & 172 & $(52.3 \%)$ & 18 & $(36.7 \%)$ & & \\
\hline FEMALE & 188 & $(49.6 \%)$ & 157 & $(47.7 \%)$ & 31 & $(63.3 \%)$ & & \\
\hline MISSING & 1 & $(0.3 \%)$ & & & & & & \\
\hline TIME AMBULANCE REQUEST N (\%) & 378 & & 329 & & 49 & & $2,676^{+}$ & .233 \\
\hline 7:00 - 8:00 & 11 & $(2.9 \%)$ & 11 & $(3.3 \%)$ & 0 & $(0.0 \%)$ & & \\
\hline $8: 00-17: 00$ & 210 & $(55.4 \%)$ & 186 & $(56.5 \%)$ & 24 & $(49.0 \%)$ & & \\
\hline $17: 00-23: 00$ & 157 & $(41.4 \%)$ & 132 & $(40.1 \%)$ & 25 & $(51.0 \%)$ & & \\
\hline MISSING & 1 & $(0.3 \%)$ & & & & & & \\
\hline DAY OF THE WEEK N (\%) & 379 & & 330 & & 49 & & $21,605^{\#}$ & $.001 *$ \\
\hline MONDAY & 56 & $(14.8 \%)$ & 47 & $(14.2 \%)$ & 9 & $(18.4 \%)$ & & \\
\hline TUESDAY & 52 & $(13.7 \%)$ & 39 & $(11.8 \%)$ & 13 & $(26.5 \%)$ & & \\
\hline WEDNESDAY & 58 & $(15.3 \%)$ & 58 & $(17.6 \%)$ & 0 & $(0.0 \%)$ & & \\
\hline THURSDAY & 64 & $(16.9 \%)$ & 54 & $(16.4 \%)$ & 10 & $(20.4 \%)$ & & \\
\hline FRIDAY & 68 & $(17.9 \%)$ & 56 & $(17.0 \%)$ & 12 & $(24.5 \%)$ & & \\
\hline SATURDAY & 37 & $(9.8 \%)$ & 36 & $(10.9 \%)$ & 1 & $(2.0 \%)$ & & \\
\hline SUNDAY & 44 & $(11.6 \%)$ & 40 & $(12.1 \%)$ & 4 & $(8.2 \%)$ & & \\
\hline AGE IN YEARS ( $\pm S D)$ & 43.1 & $(24.8)$ & 43.0 & $(24.6)$ & 43.2 & $(26.5)$ & $\begin{array}{c}t(377)= \\
0,60^{\neq \neq}\end{array}$ & .952 \\
\hline \multicolumn{9}{|l|}{${ }^{*}$ SIGNIFICANT AT $P<0.05$} \\
\hline \multicolumn{9}{|l|}{${ }^{\#} \mathbf{X}^{2}$-TEST } \\
\hline FISHER'S EXACT TEST & & & & & & & & \\
\hline
\end{tabular}

an ambulance nurse. Of the patients that requested followup care, 52/90 (57.8\%) requested this follow-up care within 12 hours after being non-conveyed. Adding the numbers of patients who received follow up care within 12 hours, 24 hours and 48 hours' time intervals, a total of $74 / 90(82.2 \%)$ of the patient requested follow-up care within 48 hours. There was no significant association between the timeframe of the followup care request and the educational level of the ambulance care professional. The degree of suffering from health after being non-conveyed was higher in the ambulance nurse group, compared to the PA-group $\left(\mathrm{X}^{2} .=6,976, \mathrm{p}=.047\right)$.

\section{Patient experience}

There were no significant differences in how patients experienced attitude and behavior, treatment, communication and the non-conveyance decision between PAs and ambulance nurses (Extended data Appendix 2), however the lower scores ("not at all' and 'yes a little') were only reported for ambulance nurses. For attitude and behavior, patients experienced the ambulance professional as polite, felt taken seriously, and felt that the ambulance professional took enough time. For treatment, patients felt reassured, trusted the ambulance professional, felt that the ambulance professional took firm action, and that the ambulance professional payed sufficient attention to other people present (family, friends, bystanders). $8.6 \%$ of the patients experienced that their pain was not or poorly managed by the ambulance care professional. For communication, patients experienced that the ambulance professional provided enough (understandable) information about what he was doing, and patients experienced the opportunity to ask questions. However, concerning the non-conveyance decision, $9.9 \%$ of the patients experienced that the reason why they were not being conveyed was not or a little explained by the ambulance professional. 


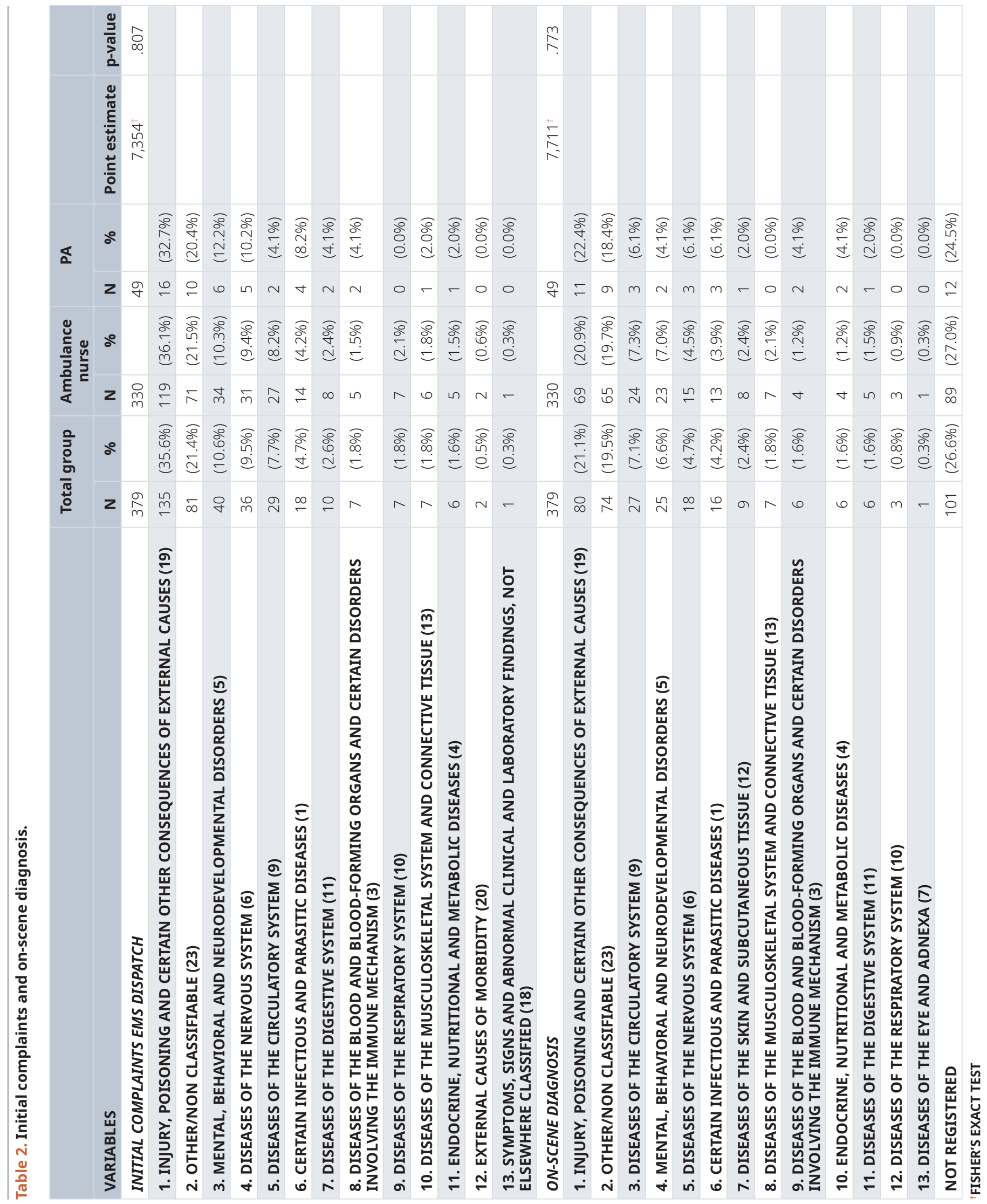


Table 3. VITAL FUNCTIONS AND OBSERVATION SCALES.

\begin{tabular}{|c|c|c|c|c|c|c|c|c|}
\hline \multirow[b]{2}{*}{ VARIABLES } & \multicolumn{2}{|c|}{ Total group } & \multicolumn{2}{|c|}{$\begin{array}{c}\text { Ambulance } \\
\text { nurse }\end{array}$} & \multicolumn{2}{|r|}{ PA } & \multirow[b]{2}{*}{$\begin{array}{c}\text { Point } \\
\text { estimate }\end{array}$} & \multirow[b]{2}{*}{ p-value } \\
\hline & $\mathbf{N}$ & $\%$ & $\mathbf{N}$ & $\%$ & $\mathbf{N}$ & $\%$ & & \\
\hline AIRWAY & 337 & & 297 & & 40 & & $0,135^{\dagger}$ & .881 \\
\hline FREE & 336 & $(99.7 \%)$ & 296 & $(99.7 \%)$ & 40 & $(100.0 \%)$ & & \\
\hline OBSTRUCTED & 1 & $(0.3 \%)$ & 1 & $(0.3 \%)$ & 0 & $(0.0 \%)$ & & \\
\hline BREATHING & 338 & & 298 & & 40 & & $8,722^{+}$ & $.038^{*}$ \\
\hline SUFFICIENT & 335 & $(99.1 \%)$ & 297 & $(99.7 \%)$ & 38 & $(95.0 \%)$ & & \\
\hline INSUFFICIENT & 3 & $(0.9 \%)$ & 1 & $(0.3 \%)$ & 2 & $(5.0 \%)$ & & \\
\hline RESPIRATORY RATE (/MIN) & 243 & & 210 & & 33 & & $0,812^{+}$ & .713 \\
\hline$<12 /$ MIN & 10 & $(4.1 \%)$ & 8 & $(3.8 \%)$ & 2 & $(6.1 \%)$ & & \\
\hline $12-20 / M I N$ & 205 & $(84.4 \%)$ & 178 & $(84.8 \%)$ & 27 & $(81.8 \%)$ & & \\
\hline$>20 / \mathrm{MIN}$ & 28 & $(11.5 \%)$ & 24 & $(11.4 \%)$ & 4 & $(12.1 \%)$ & & \\
\hline OXYGEN SATURATION & 206 & & 181 & & 25 & & $0,610^{+}$ & .348 \\
\hline$<96 \%$ & 10 & $(4.9 \%)$ & 8 & $(4.4 \%)$ & 2 & $(8.0 \%)$ & & \\
\hline $96-100 \%$ & 196 & $(95.1 \%)$ & 173 & $(95.6 \%)$ & 23 & $(92.0 \%)$ & & \\
\hline CIRCULATION & 324 & & 286 & & 38 & & $0,267^{+}$ & 1.000 \\
\hline INSUFFICIENT & 2 & $(0.6 \%)$ & 2 & $(0.7 \%)$ & 0 & $(0.0 \%)$ & & \\
\hline SUFFICIENT & 322 & $(99.4 \%)$ & 284 & $(99.3 \%)$ & 38 & $(100.0 \%)$ & & \\
\hline HEART RATE (/MIN) & 260 & & 226 & & 34 & & $1,773^{\#}$ & .206 \\
\hline$<60 / \mathrm{MIN}$ & 41 & $(15.8 \%)$ & 33 & $(14.6 \%)$ & 8 & $(23.5 \%)$ & & \\
\hline $60-100 / M I N$ & 219 & $(84.2 \%)$ & 193 & $(85.4 \%)$ & 26 & $(76.5 \%)$ & & \\
\hline HEART RHYTHM & 134 & & 117 & & 17 & & $0,064^{+}$ & .776 \\
\hline PRESENT & 36 & $(26.9 \%)$ & 31 & $(26.5 \%)$ & 5 & $(29.4 \%)$ & & \\
\hline ABSENT & 98 & $(73.1 \%)$ & 86 & $(73.5 \%)$ & 12 & $(70.6 \%)$ & & \\
\hline SYSTOLIC BLOOD PRESSURE & 189 & & 168 & & 21 & & $3,129^{+}$ & .278 \\
\hline$<90$ MMHG & 2 & $(1.1 \%)$ & 1 & $(0.6 \%)$ & 1 & $(4.8 \%)$ & & \\
\hline 90 - 160 ММнG & 161 & $(85.2 \%)$ & 143 & $(85.1 \%)$ & 18 & $(85.7 \%)$ & & \\
\hline >160 MMHG & 26 & $(13.8 \%)$ & 24 & $(14.3 \%)$ & 2 & $(9.5 \%)$ & & \\
\hline TEMPERATURE (EAR/ ${ }^{\circ} \mathrm{CELCIUS))}$ & 83 & & 68 & & 15 & & $4,317^{\dagger}$ & .098 \\
\hline$<36.1^{\circ} \mathrm{C}$ & 18 & $(21.7 \%)$ & 13 & $(19.1 \%)$ & 5 & $(33.3 \%)$ & & \\
\hline $36.1-38.0^{\circ} \mathrm{C}$ & 52 & $(62.7 \%)$ & 46 & $(67.6 \%)$ & 6 & $(40.0 \%)$ & & \\
\hline$>38.0^{\circ} \mathrm{C}$ & 13 & $(15.7 \%)$ & 9 & $(13.2 \%)$ & 4 & $(26.7 \%)$ & & \\
\hline GLASGOW COMA SCALE (EMV) & 287 & & 246 & & 41 & & $0,613^{+}$ & .431 \\
\hline$<15$ & 14 & $(4.9 \%)$ & 11 & $(4.5 \%)$ & 3 & $(7.3 \%)$ & & \\
\hline 15 & 273 & (95.1\%) & 235 & $(95.5 \%)$ & 38 & $(92.7 \%)$ & & \\
\hline AVPU & 334 & & 294 & & 40 & & $0,157^{\dagger}$ & 1.000 \\
\hline VERBAL, PAIN, UNRESPONSIVE & 12 & $(3.6 \%)$ & 11 & $(3.7 \%)$ & 1 & $(2.5 \%)$ & & \\
\hline
\end{tabular}




\begin{tabular}{|c|c|c|c|c|c|c|c|c|}
\hline \multirow[b]{2}{*}{ VARIABLES } & \multicolumn{2}{|c|}{ Total group } & \multicolumn{2}{|c|}{$\begin{array}{c}\text { Ambulance } \\
\text { nurse }\end{array}$} & \multicolumn{2}{|r|}{ PA } & \multirow[b]{2}{*}{$\begin{array}{l}\text { Point } \\
\text { estimate }\end{array}$} & \multirow[b]{2}{*}{ p-value } \\
\hline & $\mathbf{N}$ & $\%$ & $\mathbf{N}$ & $\%$ & $\mathbf{N}$ & $\%$ & & \\
\hline ALERT & 322 & $(96.4 \%)$ & 283 & $(96.3 \%)$ & 39 & $(97.5 \%)$ & & \\
\hline PUPILLARY RESPONSE & 206 & & 187 & & 19 & & $4,011^{\dagger}$ & .176 \\
\hline UNEQUAL AND NON-REACTIVE TO LIGHT & 2 & $(1.0 \%)$ & 1 & $(0.5 \%)$ & 1 & $(5.3 \%)$ & & \\
\hline EQUAL AND REACTIVE TO LIGHT & 204 & $(99.0 \%)$ & 186 & $(99.5 \%)$ & 18 & $(94.7 \%)$ & & \\
\hline PERIOD OF UNCONSCIOUSNESS & 334 & & 294 & & 40 & & $0,157^{+}$ & 1.000 \\
\hline (PERIOD) UNCONSCIOUSNESS & 12 & $(3.6 \%)$ & 11 & $(3.7 \%)$ & 1 & $(2.5 \%)$ & & \\
\hline NORMAL CONSCIOUSNESS & 322 & $(96.4 \%)$ & 283 & $(96.3 \%)$ & 39 & $(97.5 \%)$ & & \\
\hline BLOOD GLUCOSE LEVEL & 77 & & 65 & & 12 & & $1,456^{+}$ & .510 \\
\hline$<3.5 \mathrm{MMOL} / \mathrm{L}$ & 7 & $(9.1 \%)$ & 5 & $(7.7 \%)$ & 2 & $(16.7 \%)$ & & \\
\hline 3.5 - $14.0 \mathrm{MMOL} / \mathrm{L}$ & 68 & $(88.3 \%)$ & 58 & $(89.2 \%)$ & 10 & $(83.3 \%)$ & & \\
\hline$>14.0 \mathrm{MMOL} / \mathrm{L}$ & 2 & $(2.6 \%)$ & 2 & $(3.1 \%)$ & 0 & $(0.0 \%)$ & & \\
\hline REVISED TRAUMA SCORE (RTS) & 131 & & 112 & & 19 & & $2,097^{\dagger}$ & .160 \\
\hline$<12$ & 10 & $(7.6 \%)$ & 7 & $(6.3 \%)$ & 3 & $(15.8 \%)$ & & \\
\hline 12 & 121 & $(92.4 \%)$ & 105 & $(93.8 \%)$ & 16 & $(84.2 \%)$ & & \\
\hline PAIN (NUMERIC RATING SCALE) & 27 & & 23 & & 4 & & $0,181^{+}$ & 1.000 \\
\hline $4-10$ & 1 & $(3.7 \%)$ & 1 & $(4.3 \%)$ & 0 & $(0.0 \%)$ & & \\
\hline$<4$ & 26 & $(96.3 \%)$ & 22 & $(95.7 \%)$ & 4 & $(100.0 \%)$ & & \\
\hline \multicolumn{9}{|l|}{ "SIGNIFICANT AT P<0.05 } \\
\hline \multicolumn{9}{|l|}{ " $\mathbf{X}^{2}$-TEST } \\
\hline FISHER'S EXACT TEST & & & & & & & & \\
\hline
\end{tabular}

Table 4. Follow-up.

\begin{tabular}{|c|c|c|c|c|c|c|c|c|c|}
\hline \multirow[b]{2}{*}{ VARIABLES } & \multicolumn{2}{|c|}{ Total group } & \multicolumn{2}{|c|}{$\begin{array}{l}\text { Ambulance } \\
\text { nurse }\end{array}$} & \multicolumn{2}{|r|}{ PA } & \multirow[b]{2}{*}{$\begin{array}{c}\text { Point } \\
\text { estimate }\end{array}$} & \multirow[b]{2}{*}{$\begin{array}{c}p- \\
\text { value }\end{array}$} & \multirow[b]{2}{*}{ 95\%CI Difference } \\
\hline & $\mathbf{N}$ & $\%$ & $\mathbf{N}$ & $\%$ & $\mathbf{N}$ & $\%$ & & & \\
\hline REQUEST FOR FOLLOW-UP CARE & 165 & & 147 & & 18 & & & & \\
\hline YES & 90 & $(54.5 \%)$ & 80 & $(54.4 \%)$ & 10 & $(55.6 \%)$ & $5,328^{+}$ & .293 & $1.13(-24.41-24.72)$ \\
\hline YES, WITHIN 12 HOURS & 52 & $(57.8 \%)$ & 46 & $(57.5 \%)$ & 6 & $(60 \%)$ & & & \\
\hline YES, WITHIN 12 - 24 HOURS & 6 & $(6.7 \%)$ & 6 & $(7.5 \%)$ & 0 & $(0.0 \%)$ & & & \\
\hline YES, WITHIN 24 - 48 HOURS & 16 & $(17.8 \%)$ & 15 & $(18.9 \%)$ & 1 & $(10 \%)$ & & & \\
\hline YES, WITHIN 48 - 72 HOURS & 8 & $(8.9 \%)$ & 5 & $(6.3 \%)$ & 3 & $(30 \%)$ & & & \\
\hline YES, WITHIN 4 TO 7 DAYS & 8 & $(8.9 \%)$ & 8 & $(10 \%)$ & 0 & $(0.0 \%)$ & & & \\
\hline
\end{tabular}




\begin{tabular}{|c|c|c|c|c|c|c|c|c|c|}
\hline \multirow[b]{2}{*}{ VARIABLES } & \multicolumn{2}{|c|}{ Total group } & \multicolumn{2}{|c|}{$\begin{array}{l}\text { Ambulance } \\
\text { nurse }\end{array}$} & \multicolumn{2}{|r|}{ PA } & \multirow[b]{2}{*}{$\begin{array}{l}\text { Point } \\
\text { estimate }\end{array}$} & \multirow[b]{2}{*}{$\begin{array}{c}\mathrm{p}- \\
\text { value }\end{array}$} & \multirow[b]{2}{*}{ 95\%CI Difference } \\
\hline & $\mathbf{N}$ & $\%$ & $\mathbf{N}$ & $\%$ & $\mathbf{N}$ & $\%$ & & & \\
\hline TYPE OF FOLLOW-UP CARE & 90 & & 80 & & 10 & & $3,190^{+}$ & .644 & $12.5(-23.78-37.26)$ \\
\hline EMERGENCY & 37 & $(41.1 \%)$ & 34 & $(42.5 \%)$ & 3 & $(30.0 \%)$ & & & \\
\hline AMBULANCE & 3 & $(8.1 \%)$ & 3 & $(8.8 \%)$ & 0 & $(0.0 \%)$ & & & \\
\hline OUT-OF-HOURS PRIMARY CARE & 11 & $(29.7 \%)$ & 9 & $(26.5 \%)$ & 2 & $66.7 \%)$ & & & \\
\hline EMERGENCY DEPARTMENT & 23 & $(62.2 \%)$ & 22 & $(64.7 \%)$ & 1 & $(33.3 \%)$ & & & \\
\hline NON-EMERGENCY & 53 & $(58.9 \%)$ & 46 & $(57.5 \%)$ & 7 & $(70 \%)$ & & & \\
\hline OWN GENERAL PRACTITIONER & 49 & $(92.5 \%)$ & 42 & $(91.3 \%)$ & 7 & $(100 \%)$ & & & \\
\hline MENTAL HEALTH CARE & 1 & $(1.9 \%)$ & 1 & $(2.2 \%)$ & 0 & $(0.0 \%)$ & & & \\
\hline OTHER & 3 & $(5.7 \%)$ & 3 & $(6.5 \%)$ & 0 & $(0.0 \%)$ & & & \\
\hline $\begin{array}{l}\text { RELATION BETWEEN INITIAL COMPLAINS } \\
\text { AND FOLLOW-UP CARE REQUEST }\end{array}$ & 90 & & 80 & & 10 & & $0,922^{+}$ & .626 & $12.5(-11.82-37.46)$ \\
\hline YES & 82 & $(91.1 \%)$ & 73 & $(91.3 \%)$ & 9 & $(90 \%)$ & & & \\
\hline NO & 2 & $(2.2 \%)$ & 2 & $(2.5 \%)$ & 0 & $(0.0 \%)$ & & & \\
\hline I DON 'T KNOW & 6 & $(6.7 \%)$ & 5 & $(6.3 \%)$ & 1 & $(10 \%)$ & & & \\
\hline $\begin{array}{l}\text { DEGREE OF SUFFERING FROM HEALTH } \\
\text { AFTER NON-CONVEYANCE }\end{array}$ & 90 & & 80 & & 10 & & $6,976^{+}$ & $.047^{*}$ & $32.5(4.79-65.43)$ \\
\hline NOT AT ALL & 10 & $(11.1 \%)$ & 6 & $(7.5 \%)$ & 4 & $(40 \%)$ & & & \\
\hline A LITTLE BIT & 33 & $(36.7 \%)$ & 30 & $(37.5 \%)$ & 3 & $(30 \%)$ & & & \\
\hline A LOT & 32 & $(35.6 \%)$ & 30 & $(37.5 \%)$ & 2 & $(20 \%)$ & & & \\
\hline VERY MUCH & 15 & $(16.7 \%)$ & 14 & $(17.5 \%)$ & 1 & $(10 \%)$ & & & \\
\hline REASON FOLLOW-UP CARE REQUEST & 90 & & 80 & & 10 & & $1,637^{+}$ & .937 & $12.5(-27.49-35.53)$ \\
\hline ADVISED, CHECK APPOINTMENT & 35 & (38.9\%) & 31 & $(38.8 \%)$ & 4 & $(40 \%)$ & & & \\
\hline ADVISED, URGENT APPOINTMENT & 24 & $(26.7 \%)$ & 22 & $(27.5 \%)$ & 2 & $(20 \%)$ & & & \\
\hline OWN INITIATIVE, CHECK APPOINTMENT & 12 & $(13.3 \%)$ & 10 & $(12.5 \%)$ & 2 & $(20 \%)$ & & & \\
\hline $\begin{array}{l}\text { OWN INITIATIVE, URGENT } \\
\text { APPOINTMENT }\end{array}$ & 18 & $(20 \%)$ & 16 & $(20 \%)$ & 2 & $(20 \%)$ & & & \\
\hline OTHER & 1 & $(1.1 \%)$ & 1 & $(1.3 \%)$ & 0 & $(0.0 \%)$ & & & \\
\hline $\begin{array}{l}\text { MEDICATION PRESCRIPTION AFTER } \\
\text { AMBULANCE ATTENDANCE }\end{array}$ & 90 & & 80 & & 10 & & & & \\
\hline YES & 36 & $(40 \%)$ & 33 & $(41.3 \%)$ & 3 & $(30 \%)$ & $0.469^{+}$ & .734 & $11.25(-25-36.02)$ \\
\hline NO & 54 & $(60 \%)$ & 47 & (58.78\%) & 7 & $(70 \%)$ & & & \\
\hline
\end{tabular}

"SIGNIFICANT AT P $<0.05$

\# $X^{2}$-TEST

${ }^{\dagger}$ FISHER'S EXACT TEST

\section{Discussion}

The aim of this study was to compare the ambulance care process, follow-up care, and patient experience between physician assistants and ambulance nurses operating as solo ambulance care providers, for a non-conveyed patient population. It showed parity between ambulance care provided by physician assistants and ambulance nurses, but showed differences in gender, days of the week, insufficiency of breathing, and degree of suffering. Also, the study indicated that pain management and explanation about the non-conveyance decision could be improved for non-conveyed patients.

The population in this study was comparable to other studies on non-conveyance with regard to distribution of gender 
and week days, although the patients in our study were slightly younger ${ }^{14,19,26,27}$. The differences in distribution across the week could be explained by workforce scheduling by the EMS. The initial complaints during dispatch did not differ between the PA and ambulance nurse group, and are partly comparable with a previous study reporting fall/fall of height, traffic accidents, overdoses/poisoning as EMS initial complaints ${ }^{26}$. There were no significant differences between the PA and ambulance nurse groups for on-scene diagnosis, and these diagnoses were comparable with previous studies on non-conveyance ${ }^{14,19,26,27}$. Our results indicate that PAs and ambulance nurses come to comparable diagnoses after being dispatched to a group with homogenous initial complaints during dispatch. The statistically significant difference found for insufficient breathing might be due to more advanced clinical reasoning skills of the PA compared to the ambulance nurses.

In this study $54.5 \%$ of the patients requested follow-up care after being non-conveyed by the solo ambulance care provider, with no significant differences in follow-up rates between selfreferred or professional referred between the patients seen by the PA or an ambulance nurse. Although there was no significant association between type of follow-up care and educational level of the ambulance care professional, $91.9 \%$ of the follow-up emergency care requests in this study came from patients treated by an ambulance nurse, although another study showed comparable follow-up emergency care requests by PAs and ambulance nurses ${ }^{14}$. A possible explanation for this difference might be that a PA has more focus on the organ tract system examination due to their different educational background, resulting in more accurate diagnosis or treatment. Furthermore, in our study $57.8 \%$ requested follow-up care within 12 hours, and $82.2 \%$ requested follow-up care within 48 hours after being non-conveyed. To measure quality of nonconveyance within the EMS system, a follow-up indicator to measure the proportion of new healthcare contacts with time intervals at $24 \mathrm{~h}, 48 \mathrm{~h}, 72 \mathrm{~h}$ and one week would be required. Furthermore, differentiation can be made for follow-up contacts as advised by the ambulance professional, or for new or recurrent complaints.

Overall patient experiences of attitude and behavior, treatment, communication, and the non-conveyance decision by the solo ambulance professional were good, which is comparable with the literature ${ }^{13}$. From the patient perspective, there was room for improvement in the management of pain and the explanation of the non-conveyance decision. The suboptimal management of pain in EMS context has been reported previously $^{28}$. In this study, it might also explain the differences in degree of suffering between patients treated by an ambulance nurse compared to the PA. Possibly, the explanation of the non-conveyance decision to the patient might be suboptimal, as the decision is perceived as complex, and ambulance professionals experience a lack of education regarding nonconveyance assessments and decisions ${ }^{10,11}$. Also, patients experience fear as prominent emotion, which might make it more difficult for them to understand and remember a provided explanation of the non-conveyance decision ${ }^{12}$. As the proportion of non-conveyance in EMS systems is substantial and increasing, it is important to improve communication about the non-conveyance decision. This urges the need to develop educational programs not only aimed at making safe nonconveyance decisions, but also teaching how to communicate this with the patient and significant others through the concept of shared decision making. Furthermore, patients often expect conveyance while requesting ambulance care ${ }^{10}$. This addresses the need to inform patients and manage their expectations when calling an ambulance.

Besides the small statistical but not clinically significant differences in gender, days of the week, insufficiency of breathing, and degree of suffering, the care process, follow-up care, and patient experience were comparable between the ambulance nurse and PA. This might possibly be explained by the high educational level of ambulance nurses in the Netherlands, and the lack of clear task descriptions and national EMS standards for PAs in ambulance care. Therefore, PAs are not fully able to apply all medical and diagnostic skills (like drug prescription) during their professional practice, which might make a difference in prehospital care. Additionally, the effect of the PA on quality of ambulance care should be further assessed. Future research on the impact of the PA within ambulance care should be assessed by using the six dimensions of quality of healthcare: (1) effectiveness, (2) efficiency, (3) patient safety, (4) accessibility, (5) timeliness and (6) target population directed ${ }^{15}$. The effects on these dimensions should be measured in all phases of the ambulance process, from initial call and dispatch triage, to handover or referral. Also, the implementation of the PA should be investigated, by gaining in-depth insight in factors influencing this implementation process from the perspective of the patient, the professional, the organization, and chain of emergency care.

\section{Limitations}

Despite the fact that this study is one of the first reporting on the quality of care of PAs in ambulance care, this study had several limitations. The first limitation is the partial use of retrospective data and the relatively small sample size for the patients seen by a PA, which means that the results could be biased by a lack of power. For future research to test differences in follow-up care after non-conveyance, we calculated a sample size estimation in order to achieve more statistical power. Based on a $95 \%$ confidence interval, a non-conveyed population size of $10,000-15,000^{27}$ and a $5 \%$ margin of error, a sample size of 370-375 would be advisable for future studies. Secondly, the questionnaire used was developed based on a validated instrument and literature, but was not additionally validated for this study. Thirdly, due to random suboptimal registration we had missing data on all variables, especially vital functions/observation scales. Missing data in nonconveyance studies has been described earlier ${ }^{19}$. Finally, the Netherlands has a specific EMS-system and this might limit generalizability to other healthcare systems.

\section{Conclusion}

Besides small statistical but not clinically significant differences, this study indicated similarity in solo ambulance care provided by a physician assistant or an ambulance nurse concerning the care process, follow-up care, and patient experience. 
Regarding the type of follow-up care, patients treated by a PA seek less emergency care after being non-conveyed, compared to an ambulance nurse. Overall, patients experienced good attitude and behavior, treatment, and communication from the solo ambulance professional, with no differences between the PAs and ambulance nurses. From the patient's perspective, pain management and explanation about the non-conveyance decision could be improved. Future well-powered studies to gain insight in effects of PAs in ambulance care are needed, as well as studies in which PAs apply all additional skills they are licensed to.

\section{Data availability statement}

Underlying data

DANS-EASY: Underlying data for 'Quality of solo ambulance care by physician assistants versus ambulance nurses for non-conveyed patients in the Netherlands: An observational study' https://doi.org/10.17026/dans-zqw-qbqp ${ }^{29}$.

This project contains the following underlying data:

- Anonomised dataset (title: 20210407 Anonymized dataset.sav)

\section{- $\quad$ STROBE-statement}

Data are available under the terms of the Creative Commons Zero "No rights reserved" data waiver (CC0 1.0 Universal (CC0 1.0) Public Domain Dedication).

\section{Extended data}

DANS-EASY: Underlying data for 'Quality of solo ambulance care by physician assistants versus ambulance nurses for nonconveyed patients in the Netherlands: An observational study' https://doi.org/10.17026/dans-zqw-qbqp ${ }^{29}$.
This project contains the following extended data:

- $\quad$ Appendix 1

- $\quad$ Appendix 2

Extended data are available under the terms of the Creative Commons Zero "No rights reserved" data waiver (CC0 1.0 Universal (CC0 1.0) Public Domain Dedication).

\section{Ethical approval}

The Ethical Research Committee of the HAN University of Applied Sciences critically appraised the research protocol and concluded that the study complied with the criteria of the Declaration of Helsinki on Ethical Principles for Medical Research Involving Human Subjects, applicable national laws (like the General Data Protection Regulation), and the Dutch code of conduct for Research Integrity (reference Ethical Research Committee HAN University of Applied Sciences: 241.02/21).

\section{Consent statement}

Patient were given an information letter alongside their questionnaire, which stated that if the patient returned the questionnaire, this was interpreted as informed consent to participate. In this way written informed consent for publication of the patients' details was obtained from the patients.

\section{Acknowledgements}

The authors would like to thank Steven Teerenstra $\mathrm{PhD}$. for his statistical advise during data-analysis, and Rianne van Boekel PhD and Paul JT Rood RN PhD for their feedback during the writing process.
1. Lowthian JA, Cameron PA, Stoelwinder JU, et al: Increasing utilisation of emergency ambulances. Aust Health Rev. 2011; 35(1): 63-69. PubMed Abstract | Publisher Full Text

2. Christensen EF, Larsen TM, Jensen FB, et al: Diagnosis and mortality in prehospital emergency patients transported to hospital: a populationbased and registry-based cohort study. BMJ Open. 2016; 6(7): e011558. PubMed Abstract | Publisher Full Text | Free Full Text

3. Edwards MJ, Bassett $G$, Sinden $L$, et al.: Frequent callers to the ambulance service: patient profiling and impact of case management on patient utilisation of the ambulance service. Emerg Med J. 2015; 32(5): 392-396. PubMed Abstract | Publisher Full Text

4. Søvsø, MB, Kløjgaard TA, Hansen PA, et al:: Repeated ambulance use is associated with chronic diseases - a population-based historic cohort study of patients' symptoms and diagnoses. Scand I Trauma Resusc Emerg Med 2019; 27(1): 46

PubMed Abstract | Publisher Full Text | Free Full Text

5. Booker MJ, Shaw ARG, Purdy S: Why do patients with 'primary care sensitive' problems access ambulance services? A systematic mapping review of the literature. BMJ Open. 2015; 5(5): e007726. Publisher Full Text

6. Jones CMC, Wasserman EB, Li T, et al.: The Effect of Older Age on EMS Use for Transportation to an Emergency Department. Prehosp Disaster Med. 2017, 32(3): 261-268.

PubMed Abstract | Publisher Full Text
7. Cone DC, Kim DT, Davidson SJ: Patient-initiated refusals of prehospital care: ambulance call report documentation, patient outcome, and on-line medical command. Prehosp Disaster Med. 1995; 10(1): 3-9.

PubMed Abstract | Publisher Full Text

8. Larsen T, Bendtsen M, Søvsø M, et al.: Treat-and-release EMS patients in the north denmark region: identification and vital signs. BMJ Open. 2017; 7: A13. Publisher Full Text

9. Ebben RHA, Vloet LCM, Speijers RF, et al.: A patient-safety and professional perspective on non-conveyance in ambulance care: a systematic review. Scand J Trauma Resusc Emerg Med. 2017; 25(1): 71. PubMed Abstract | Publisher Full Text | Free Full Text

10. Lederman J, Löfvenmark C, Djärv T, et al:: Assessing non-conveyed patients in the ambulance service: a phenomenological interview study with Swedish ambulance clinicians. BMJ Open. 2019; 9(9): e030203. PubMed Abstract | Publisher Full Text | Free Full Text

11. Höglund $E$, Schröder $A$, Möller $M$, et al.: The ambulance nurse experiences of non-conveying patients. J Clin Nurs. 2019; 28(1-2): 235-244. PubMed Abstract | Publisher Full Text | Free Full Text

12. van Doorn SCM, Verhalle RC, Ebben RHA, et al:: The experience of nonconveyance following emergency medical service triage from the perspective of patients and their relatives: A qualitative study. Int Emerg Nurs. 2021; 54: 100952. PubMed Abstract | Publisher Full Text 
13. King R, Oprescu F, Lord B, et al.: Patient experience of non-conveyance following emergency ambulance service response: A scoping review of the literature. Australas Emerg Care. 2020; S2588-994X(20)30083-X. PubMed Abstract | Publisher Full Text

14. Bloemhoff A, Schoonhoven L, de Kreek AJ, et al.: Solo emergency care by a physician assistant versus an ambulance nurse: a cross-sectional document study. Scand J Trauma Resusc Emerg Med. 2016; 24: 86. PubMed Abstract | Publisher Full Text | Free Full Text

15. van Vliet $R$, Ebben $R$, Diets $N$, et al.: Nurse practitioners and physician assistants working in ambulance care: A systematic review [version 1; peer review: 1 approved, 2 approved with reservations]. F1000Res. 2020; 9: 1182. PubMed Abstract | Publisher Full Text | Free Full Text

16. Doan Q, Sabhaney V, Kissoon N, et al:: A systematic review: The role and impact of the physician assistant in the emergency department. Emerg Med Australas. 2011; 23(1): 7-15. PubMed Abstract | Publisher Full Text

17. von Elm E, Altman DG, Egger M, et al.: Strengthening the Reporting of observational Studies in Epidemiology (STROBE) statement: guidelines for reporting observational studies. BMJ. 2007; 335(7624): 806-808. Publisher Full Text

18. Carlström E, Fredén $\mathrm{L}$ : The first single responders in Sweden - Evaluation of a pre-hospital single staffed unit. Int Emerg Nurs. 2017; 32: 15-19. PubMed Abstract | Publisher Full Text

19. Breeman W, Poublon NA, Verhofstad MHJ, et al:: Safety of on-scene medical care by EMS nurses in non-transported patients: a prospective, observational study. Scand J Trauma Resusc Emerg Med. 2018; 26(1): 79. Publisher Full Text

20. Ebben $\mathrm{RH}$, Vloet LC, Schalk DM, et al.: An Exploration of Factors Influencing Ambulance and Emergency Nurses' Protocol Adherence in the Netherlands. J Emerg Nurs. 2014; 40(2): 124-30 PubMed Abstract | Publisher Full Text

21. Lovink $\mathrm{MH}$, Persoon A, van Vught AJAH, et al.: Substituting physicians with nurse practitioners, physician assistants or nurses in nursing homes: protocol for a realist evaluation case study. BMJ Open. 2017; 7(6): e015134. PubMed Abstract | Publisher Full Text | Free Full Text

22. Drijver CR: [Use the Netherlands Triage Standard for children]. Ned Tijdschr Geneeskd. 2015; 159: A8330.

PubMed Abstract

23. WHO Library Cataloguing-in-Publication Data:International statistical classification of diseases and related health problems. - 10th revision, edition 2010. 2011 Reference Source

24. Van de Ven D, Bos N, De Boer D: Kwaliteit van ambulancezorg vanuit het perspectief van cliënten: ervaringen van cliënten met de ambulancezorg gemeten met de CQ-indexen planbare en spoedeisende ambulancezorg. 2017. Reference Source

25. Krol M, Sixma H, Plass AM: CQI Spoedeisende Ambulancezorg: actualisatie en bepaling van het discriminerend vermogen. 2013: 1. Reference Source

26. Ebben RHA, Castelijns M, Frenken J, et al:: Characteristics of non-conveyance ambulance runs: A retrospective study in the Netherlands. World J Emerg Med. 2019; 10(4): 239-243.

PubMed Abstract | Publisher Full Text | Free Full Text

27. Vloet LCM, de Kreek A, van der Linden EMC, et al.: A retrospective comparison between non-conveyed and conveyed patients in ambulance care. Scand J Trauma Resusc Emerg Med. 2018; 26(1): 91. PubMed Abstract | Publisher Full Text | Free Full Text

28. Berben SA, Schoonhoven L, Meijs TH, et al.: Prevalence and relief of pain in trauma patients in emergency medical services. Clin J Pain. 2011; 27(7): 587-592.

PubMed Abstract | Publisher Full Text

29. Ebben RHA: Quality of solo ambulance care by physician assistants versus ambulance nurses for non-conveyed patients: An observational study in the Netherlands. DANS. 2021.

http://www.doi.org/10.17026/dans-zqw-qbqp 


\section{Open Peer Review}

\section{Current Peer Review Status: ?}

\section{Version 1}

Reviewer Report 16 January 2023

https://doi.org/10.5256/f1000research.54940.r157722

(C) 2023 Frischknecht Christensen E. This is an open access peer review report distributed under the terms of the Creative Commons Attribution License, which permits unrestricted use, distribution, and reproduction in any medium, provided the original work is properly cited.

\section{Erika Frischknecht Christensen}

Clinic of Internal and Emergency Medicine, Department of Emergency and Trauma Care, Aalborg University Hospital, Aalborg, Denmark

Thanks for letting me review this paper about care made by physician assistants (PA) vs ambulance nurses in non-conveyance of EMS patients. The topic is of interest in emergency care as new strategies are need and should be investigated. However, physician assistant (PA) is not a well-known category within EMS and the introduction can be improved by explaining this to the reader and how PA's are expected to improve or change care. Is a solo ambulance like other ambulances for transporting patients or is it simply a car? And if it is an ambulance does the PA alone take the patient onto the trolley in the conveyed cases? Also, the international reader needs information about the Dutch Triage Standard - and how complaints can be converted to ICD-10 diagnoses (table 2)

The study aims are ambitious with several outcomes and follow up. All-over, the data material seems sound with EMS patient data sources and questionnaires. The main methodological limitation is the small sample size: the rather low number of patients, especially the low number of PA cases and the low response rate: 1549 solo-ambulances out of 40910 ambulances (2-3\%), and only 379 of these solo-ambulances included, with PA in 49 (13\%) cases - and follow-up on 165 (44\%), and among these only 18 PA. This is contrasting the large number of factors (time of the day, the week, complaints, diagnoses, vital functions (14 factors) analyzed and compared, implying the risk both of type II error due to the low number (as discussed by the authors) and risk of 'multi-significance' - finding statistically significant differences just by chance. I recommend focusing on the few most important factors and outcomes. Missing data were treated as random in the analyses but only partly reported in the tables. What are the possible implications of missing data?

The follow-up by questionnaires is interesting, and showed that more than half of the patients requested follow-up (and of these 52/90 (58\%) within 12 hours) which seems high and may indicate that non-conveyance was not the best decision? Also, half of the respondents reported a lot or very much suffering. There might be a bias due the low response rate, or? Please, discuss. 
It is reported, both in results, conclusion and abstract that $91.9 \%$ of the follow-up was in the ambulance nurse group - based on which calculations? Seems to correspond to the distribution of 80 ambulance nurses and only 10 PA in the follow-up analyses. Please be very cautious, both in reporting results and in the discussion and conclusions based on the follow-up data.

It is appreciated that the patients were asked about their experience, but I did not get access to the appendix.

Finally, considering the concept of non-conveyance, this study seems to indicate that benefit is almost exclusively for EMS, as only $8 \%$ needed an ambulance, while two thirds had follow-up in the ED and almost one third in out-of-hours primary care. Reflections?

Is the work clearly and accurately presented and does it cite the current literature? Partly

Is the study design appropriate and is the work technically sound? Partly

Are sufficient details of methods and analysis provided to allow replication by others? Partly

If applicable, is the statistical analysis and its interpretation appropriate? Partly

Are all the source data underlying the results available to ensure full reproducibility? No source data required

Are the conclusions drawn adequately supported by the results? Partly

Competing Interests: No competing interests were disclosed.

I confirm that I have read this submission and believe that I have an appropriate level of expertise to confirm that it is of an acceptable scientific standard, however I have significant reservations, as outlined above. 
The benefits of publishing with F1000Research:

- Your article is published within days, with no editorial bias

- You can publish traditional articles, null/negative results, case reports, data notes and more

- The peer review process is transparent and collaborative

- Your article is indexed in PubMed after passing peer review

- Dedicated customer support at every stage

For pre-submission enquiries, contact research@f1000.com 